\title{
High-resolution computed microtomography for the characterization of a diffusion tensor imaging phantom
}

\author{
Lukasz Kaczmarek $^{1,2}$ (1) $\cdot$ Tomasz Wejrzanowski ${ }^{2} \cdot$ Jakub Skibiński $^{2} \cdot$ \\ Michał Maksimczuk ${ }^{2} \cdot$ Artur Krzyżak $^{3}$
}

Received: 26 December 2016/ Accepted: 17 February 2017/Published online: 6 March 2017

(c) The Author(s) 2017. This article is published with open access at Springerlink.com

\begin{abstract}
This paper addresses the issue of the quantitative characterization of the structure of the calibration model (phantom) for b-matrix spatial distribution diffusion tensor imaging (BSD-DTI) scanners. The aim of this study was to verify manufacturing assumptions of the structure of materials, since phantoms are used for BSD-DTI calibration directly after manufacturing. Visualization of the phantoms' structure was achieved through optical microscopy and high-resolution computed microtomography $(\mu \mathrm{CT})$. Using $\mu \mathrm{CT}$ images, a numerical model of the materials structure was developed for further quantitative analysis. 3D image characterization was performed to determine crucial structural parameters of the phantom: porosity, uniformity and distribution of equivalent diameter of capillary bundles. Additionally calculations of hypothetical flow streamlines were also performed based on the numerical model that was developed. The results obtained in this study can be used in the calibration of DTI-BST measurements. However, it was found that the structure of the phantom exhibits flaws and discrepancies from the assumed geometry which might affect BSD-DTI calibration.
\end{abstract}

Łukasz Kaczmarek

lukasz.kaczmarek@uw.edu.pl;

Lukasz.Kaczmarek@wimpw.edu.pl

1 Faculty of Geology, University of Warsaw, Żwirki i Wigury 93, 02-089 Warsaw, Poland

2 Faculty of Materials Science and Engineering, Warsaw University of Technology, Wołoska 141, 02-507 Warsaw, Poland

3 Faculty of Geology, Geophysics and Environmental Protection, University of Science and Technology in Kraków, Mickiewicza 30, 30-059 Kraków, Poland
Keywords Phantom · Internal structure $\cdot$ Non-destructive analysis $\cdot$ Numerical models

\section{Introduction}

BSD-DTI is one of the magnetic resonance imaging (MRI) approaches to diffusion tensor imaging, taking into consideration the actual spatial distribution of the b-matrix, which corresponds to the distribution of the magnetic field gradients (Krzyżak and Olejniczak 2014). MRI techniques are used for porous material saturation analysis and diffusion study. This technique is applied in various issues from medical investigations (e.g., Tomanek et al. 1996; Das 2004 or Krzyżak et al. 2005, 2008) to petroleum geology reservoir rock characterization (e.g., Wei et al. 2015; Xiao et al. 2013). The principles and basis of classic MRI technology were described in detail by Callaghan (1994), Straley et al. (1995) and Coates et al. (1999). Results obtained through MRI depend, to a large extent, on scanner properties, sequence parameters and characteristics of samples. To obtain accurate MRI results, the scanner needs to be properly calibrated, involving the determination of the relation of $\mathrm{T} 1, \mathrm{~T} 2$ relaxation times with sample porosity. The calibration process requires the application of phantoms: samples with a precisely defined structure (Komlosh et al. 2011). Furthermore, in BSD-DTI, welldefined anisotropic phantoms are central to improving accuracy and determining diffusion gradient directions and magnitudes (Komlosh et al. 2011). This is due to impact of the inhomogeneity of the analyzed material on the inhomogeneity of the magnetic field, which influences the relaxation processes and timing of each sequence. In general, the registered MRI signal errors of the calibration phantom are composed of two main components: (1) 
systematic errors induced by the nonlinearity of imaging gradients (e.g., diffusion gradients), and (2) imperfections of the phantom. Knowing the exact structure of the phantom, we can accurately and spatially determine its effect on the signal, and thus identify the actual distribution of imaging gradients. This can significantly reduce the influence of the heterogeneity of the magnetic field gradients for MRI experiments, particularly in DTI. In principle, BSDDTI assumes carrying out standard DTI imaging with at least six different directions of diffusion gradients (plus one without) for at least six positions of anisotropic phantom-three orthogonal, aligned with the laboratory frame axes and three oblique. The measurement results are sufficient to determine the diffusion tensor, assuming its symmetry. Due to the structural complexity of the phantoms and possible defects which may occur during the manufacturing process, the phantom obtained can differ from the designed geometry. Therefore, verification and quantitative description of phantom geometry is an essential step. Microtomography is a non-destructive tool that provides information about the internal structure of phantoms, enabling detailed analysis of the homogeneity and beam of capillaries geometry, and lending valuable insight for the development of numerical models. The theoretical basis of $\mu \mathrm{CT}$ was very well described by Ketcham and Carlson (2001), Baker et al. (2012) and by Gnudde and Boone (2013) in the review articles. Examples of $\mu \mathrm{CT}$ analysis of various types of porous media were presented in articles by Appoloni et al. (2007), Bielecki et al. (2013), Kaczmarek et al. (2015). $\mu \mathrm{CT}$ has also been used for analysis of flow through rock specimens by: Petchsingto and Karpyn (2009), Dvorkin et al. (2009). Literature data and our own investigations indicate that $\mu \mathrm{CT}$ analysis has a wide range of applications in geology.

This study presents $\mu \mathrm{CT}$ analysis of phantoms' internal structure and its parameterization after the manufacturing process. The main goal of this study was to verify the structure of the material and identify potential discrepancies that could influence the BST-DTI calibration process. Subsequently, numerical simulations of fluid flow through the phantom sample were performed to study the linearity of the capillaries and influence of flaws on the streamlines of fluid inside the phantom. The geometry and size of the internal elements of phantoms depend on the specific MRI approach. Therefore, the presented methodology is a universal solution and can be adapted for use with a variety of phantom types.

\section{Materials and methods}

The internal structure of phantoms relates to the particular research technique employed. Accordingly, the presented study procedure can be easily adopted for use with a variety of MRI scanners. For initial recognition, we used a light microscope followed by a non-destructive techniquecomputed microtomography $(\mu \mathrm{CT})$. Results obtained from $\mu \mathrm{CT}$ were visualized with commercial software (SkyScan ${ }^{\circledR}$ 1.13.11.0 and Avizo ${ }^{\circledR}$ 8.0). Figure 1 shows a schematic chart of the multistage analysis that was conducted.

\section{Sample}

The cylindrical phantom dedicated for the calibration of BST-DTI scanners was constructed by the Military University of Technology in Warsaw. More detailed information about manufacturing can be found in Krzyzak et al. (2015). The linear structure is determined by capillary bundles. This structure enables lateral free diffusion along capillaries and restricts diffusion crosswise without any distortion, a crucial condition for the improvement of accuracy and better determination of the diffusion gradient directions and, therefore, the usefulness of phantoms. Figure 2 shows the investigated cylindrical glass phantom, which was filled with distilled water. The sample was a $3.6 \mathrm{~cm}$ long cylinder with a diameter of $3.4 \mathrm{~cm}$, densely packed with hexagonal high precision acrylic glass bundles of capillaries (each with a diameter of approx. $920 \mu \mathrm{m}$; total number equal 1502). Each bundle of capillaries consisted of dozens of capillaries (each with a diameter of approx. $36 \mu \mathrm{m}$ ). The bundles of capillaries are enclosed in a glass sphere to prevent water loss.

Additionally, it should be noted that it is possible to define and manufacture various types of phantom structures and use them as models of the real structures of rocks. It is the characteristic features of the sedimentary rock structure like capillaries and lamination, which are important for flow media, as shown by Sun et al. (2015), where the relationship between the permeability, microfractures and the stresses in the rock is studied. These structural features can be reproduced in the phantoms by bundles of capillaries.

\section{Imaging}

\section{Light microscopy}

A Nikon EPIPHOT 200 was used for initial observations, providing images of the top or bottom surface of the

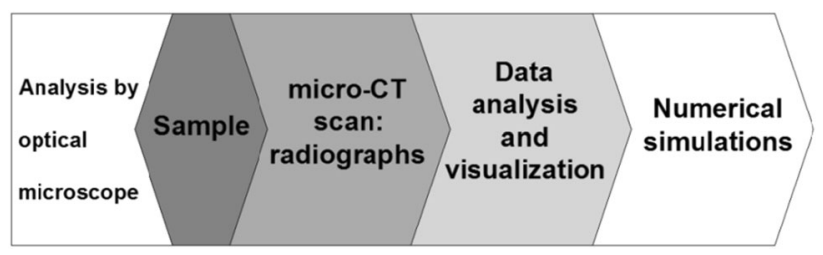

Fig. 1 Workflow of phantom analysis 


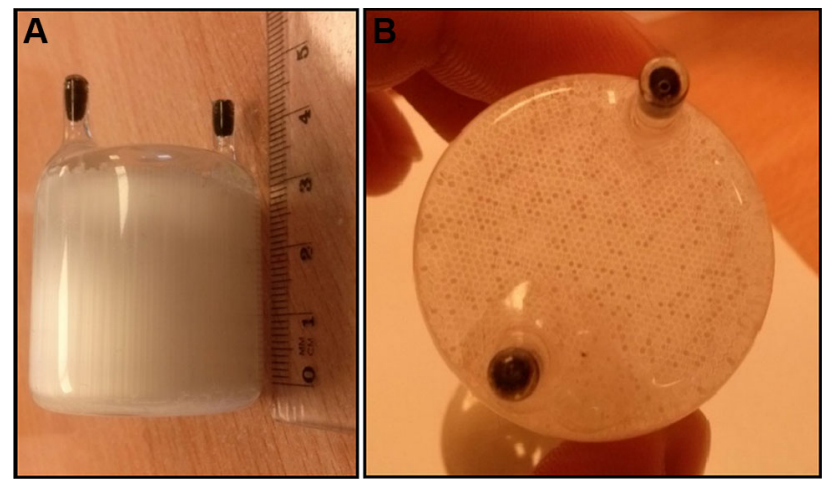

Fig. 2 a Sidewall of cylindrical phantom with visible longitudinal moving bundles of capillaries, $\mathbf{b}$ Phantom's upper surface with visible cellular cross-sections of bundles of capillaries

phantom. The advantage of this method was the ability to obtain images of the individual capillaries, through $75 \times$ and $8 \times$ visual magnification of sample. The drawback of this method was distorted and locally blurred images. Furthermore, light causes an increase in temperature of the sample during observation and longer observation can lead to the rupture of phantom glass.

\section{$X$-ray microtomography}

The main advantage of $\mu \mathrm{CT}$ is the non-destructive character of internal structure analysis. High-resolution computed microtomography is based on imaging the variable coefficient of linear absorption of the material depending on density. We can also simulate fluid flow inside the sample on the basis of a numerical model obtained from high-resolution images. The numerical model obtained from tomography images precisely represents the geometry of the studied material. Xradia Micro XCT-400 was used for the analysis. The acquisitions were performed using a Hamamatsu L8121-03, which generates X-rays in the range of $40-150 \mathrm{kV}$. The scanned image is converted to digital data using a CCD video system, which has a resolution of $1024 \times 1024$ pixels, with 16-bit image depth of the detector. Selected samples were scanned using different acquisition parameters to select the appropriate settings for the technical equipment, producing images. A large field of view (LFOV) lens with 0.5 zoom was used during tests to obtain the broadest area of recognition. The manufacturer's LE3 custom glass filters were used for beam filtration. Scanning parameters are shown in Table 1.

\section{Image processing}

During radiograph processing, noise removal processes were performed, i.e., beam hardening (0.8) and center shift (2.0). The reconstruction parameters were binning 1 with bright spots correction and smoothing (Kernel size $=0.5$ ). Then radiographs were reconstructed to obtain a set of 1024 highresolution bitmap images. Then, the images were processed from 16 bit to 8 bit to reduce the size of images for faster processing Subsequently, the set of images was used to develop a numerical model enabling visualization of the samples. After reconstruction, the thresholding method of 8-bit (256 gray scale) images was performed to distinguish phantom bundles of capillaries. Owing to $\mu \mathrm{CT}$ processing software limitations, the analysis area had to be reduced to the working area (region of interest-ROI) in the cuboid form. Additionally, it should be noted that the size of voxel is the result of the size of ROI. The sample was earmarked for further DTI studies, and so singular bundles of capillaries could not be cut off for additional $\mu \mathrm{CT}$ analysis with smaller ROI and smaller voxel size. In the study, the voxel size of the analyzed images was $11 \mu \mathrm{m}$. ROI was selected in the center of the Phantom $(13 \times 16 \times 10 \mathrm{~mm})$ and the prepared images were smoothed using a 3D median filter. A filter was used at a very low level ( 2 pixels), with the aim being to remove single pixels. The pixels present in the image were the result of the binarization. The low level of median filter, on the other hand, meant that the sharp edges of the phantom structure could be retained. Based on a binary set of image slices, a three-dimensional numerical model could be generated. A schematic chart of image preparation for numerical analysis is shown in Fig. 3.

$\mu \mathrm{CT}$ analysis revealed information regarding the incomplete adhesion of capillary bundles, which was a discrepancy from the design assumptions. Due to these defects, empty channels without capillary bundles were created. Image analysis was performed that pertains to this issue, as shown in Fig. 3.

\section{Phantom parameters}

One of the most important parameters describing the storage capacity of rock is porosity. This paper concerns the porosity parameter considered as a fraction of voxels assigned to the pore regions with regard to the total number of voxels: $V_{\mathrm{v}}=V_{\mathrm{p}} / V$ where: $V_{\mathrm{v}}$ is the porosity, $V_{\mathrm{p}}$ is the pore volume and $V$ is the total volume of the sample.

Table 1 Parameters of X-ray microtomography

\begin{tabular}{llllll}
\hline Scan number & Voltage $(\mathrm{kV})$ & Intensity $(\mu \mathrm{A})$ & Exposure time $(\mathrm{s})$ & Type of objective $(-)$ & Voxel size $(\mu \mathrm{m})$ \\
\hline 1 & 110 & 90 & 2 & LFOV & 34 \\
2 & 140 & 90 & 9 & LFOV & 11 \\
\hline
\end{tabular}




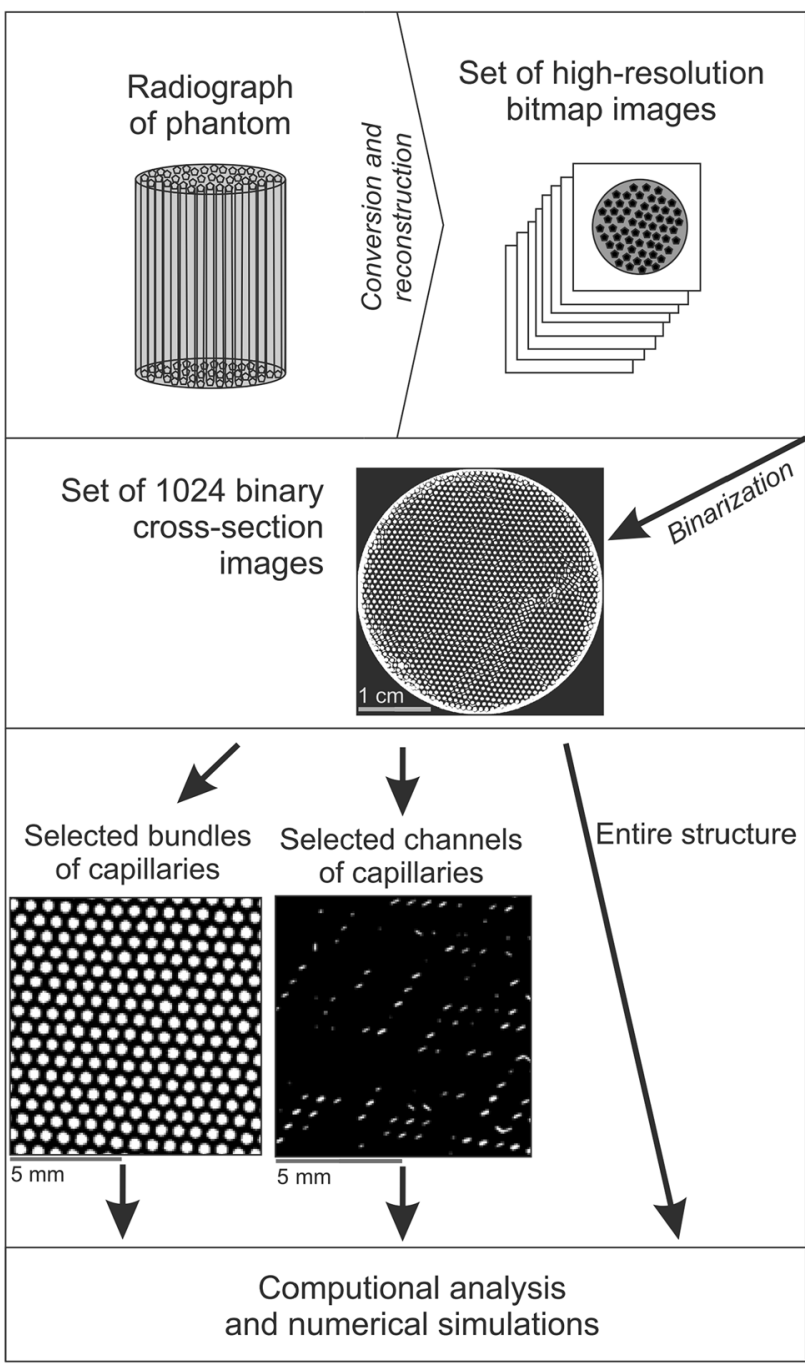

Fig. 3 Schematic chart of quantitative characterization and visualization of the phantom structure

The structure of the phantom with directional anisotropy could be described by the same parameters, based on the stereology of materials (Wejrzanowski et al. 2008). The mean values of calculated parameters used to describe the 3D internal structure of the phantom are shown in Table 2.

The homogeneity of the phantom was analyzed using Voronoi tessellation (Aurenhammer 1991). Cross-sections capillary bundles exhibit a cellular structure and, thus, the distribution of each "cell" size is expressed by the statistical parameter - coefficient of variation $\mathrm{CV}(f)$, which described the homogeneity of the studied sample.

\section{Fluid flow simulation}

Numerical simulations of fluid flow and the subsequent visualization of streamlines (flow paths) were performed using the finite volume method (FVM) employing the developed model. Based on $\mu \mathrm{CT}$ results, we created a model of the sample for application in numerical simulations of fluid flow through a phantom structure.

The continuity and Navier-Stokes equations for the flow of incompressible Newtonian fluid at steady-state can be expressed as:

$\rho u \cdot \nabla u=-\nabla p+\mu \nabla^{2} u$,

$\nabla \cdot u=0$

where $\mu$ and $\rho$ are the viscosity and density of the fluid, while $u$ and $p$ are local velocity and pressure, respectively. To solve the above equations and calculate fluid flow in the studied sample, Avizo software was employed using FVM. This method transforms partial differential equations into algebraic ones. Values are calculated at discrete places on meshed geometry.

The integral form of the conservation law can be rewritten using the Gauss divergence theorem:

$\mathrm{\partial} / \partial t \int_{\Omega} U \mathrm{~d} \Omega+\int_{\Omega} \vec{\nabla} \cdot \vec{F} \mathrm{~d} \Omega=0$,

where $U$ represents a vector of states and $F$ represents the corresponding flux tensor. The volume integrals of divergence terms in a partial differential equation are converted to surface integrals of fluxes all around the control volume (4).

$\partial / \partial t \int_{\Omega} U \mathrm{~d} \Omega+\oint_{\partial \Omega} \vec{F} \cdot \vec{n} \mathrm{~d} \Gamma=0$,

where $\Gamma$ represents the total surface area of the cell and $n$ is a unit vector normal to the surface and pointing outward. The resulting equations can be solved numerically via discretization into a set of discrete volumes which can be well defined for the porous structures in question.

In the case of further detailed analysis, it is possible to perform a digital experiment of permeability. The test is based on fluid flow simulation through a numerical model of the rock sample. Due to the previous $\mu \mathrm{CT}$ scanning of the sample, it is possible to reconstruct the exact shapes of the flow paths in the model. A similar study of permeability determination was presented by Dvorkin et al. (2009).

In this study, the flow across both designed and real structures was simulated using Avizo software with the computational domain of length $13 \times 16 \times 10 \mathrm{~mm}$ (Fig. 4). The inlet velocities for FVM simulations correspond to the Reynolds number from 1 to 10 . The Newtonian fluid flowing through the channel was oil, in accordance with the archive viscosity data of oil to mirror real flow conditions from the Baltic Basin shale formations in Poland (Twarduś and Nowicka 2014). 


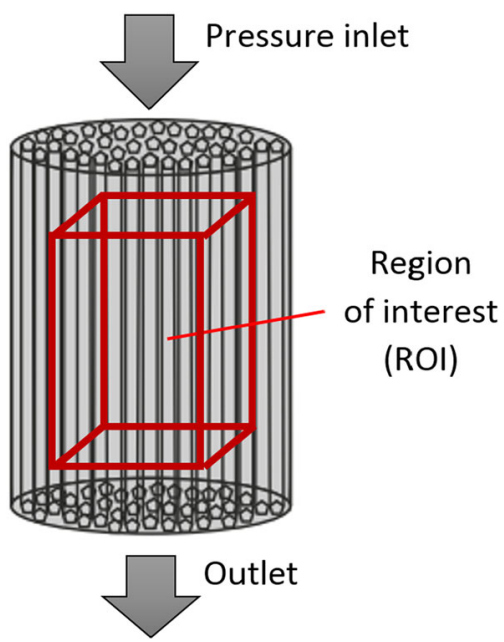

Fig. 4 Scheme of numerical simulation of flow

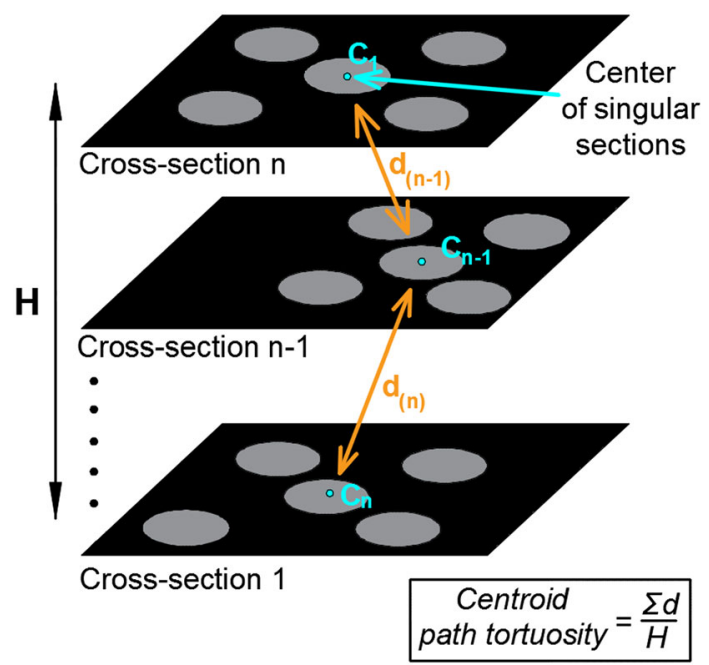

Fig. 5 Scheme of centroid path tortuosity calculation

A mesh consisting of 988,000 elements was generated based on the geometry of the studied sample, which provided an accurate representation of the internal structure without distorting generalizations. Calculations were performed to visualize streamlines, enabling qualitative verification of the linearity of the structure of capillaries and comparison with rock fissures. Furthermore, the tortuosity can also be calculated, based on the centroid path tortuosity module in AVIZO software. In reference to the software calculation procedure, this general parameter is understood as the ratio of the real length of the flow path to the straight line between the beginning and the end of flow (Fig. 5). Furthermore, Petchsingto and Karpyn (2009) have shown that tortuosity can be interpreted in relation to the pressure gradient profile of flow.

\section{Results and discussion}

\section{Light microscopy}

The glass capillary array phantom is shown in Fig. 6 as a photographic enlargement and microscopic image. The cross-section of the phantom reveals its regular internal structure. Owing to the lack of thin wall adhesion, small channels are observed among the capillary bundles. The greatest restriction on optical microscopy image analysis was the thickness of the phantom's protective casing, which resulted in image blurring.

During the first stage of the research, we could observe slight movement of the capillary bundles in the phantom. The narrow edges of the bundles peeling off from the outer casing caused this damage.

\section{X-ray microtomography and numerical simulations}

During analysis of the phantom, we observed disorders of the structure of capillary bundles. Firstly, the shifts between bundles created diversified channels (Fig. 7). Secondly, gaps between bundles were observed. Additionally, size differences were noticed during the image analysis. To accurately describe geometric structure relations, parameters were chosen that described the internal
Fig. 6 Optical microscopy image of sample: A.a. capillary bundles of cylindrical phantom; A.b. singular capillaries of cylindrical phantom
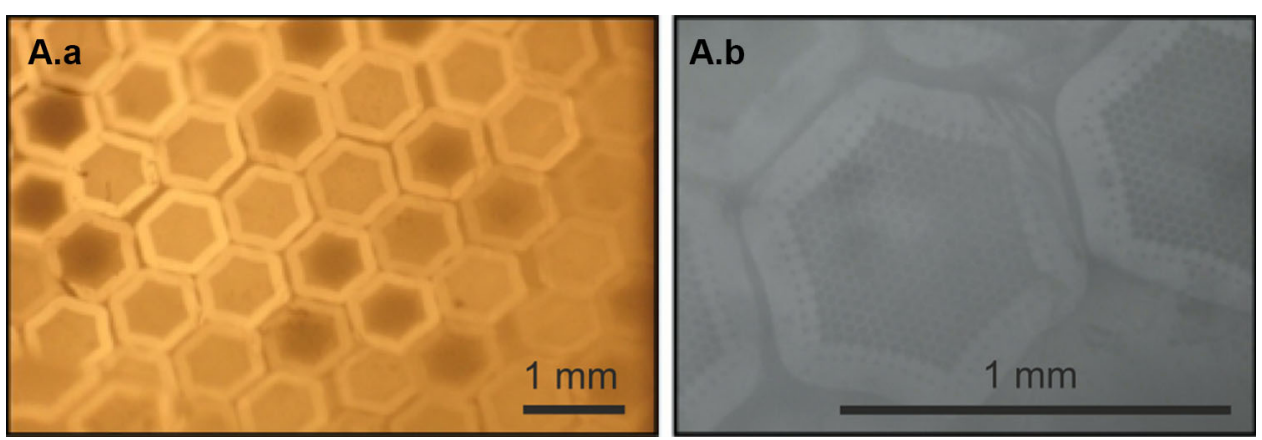


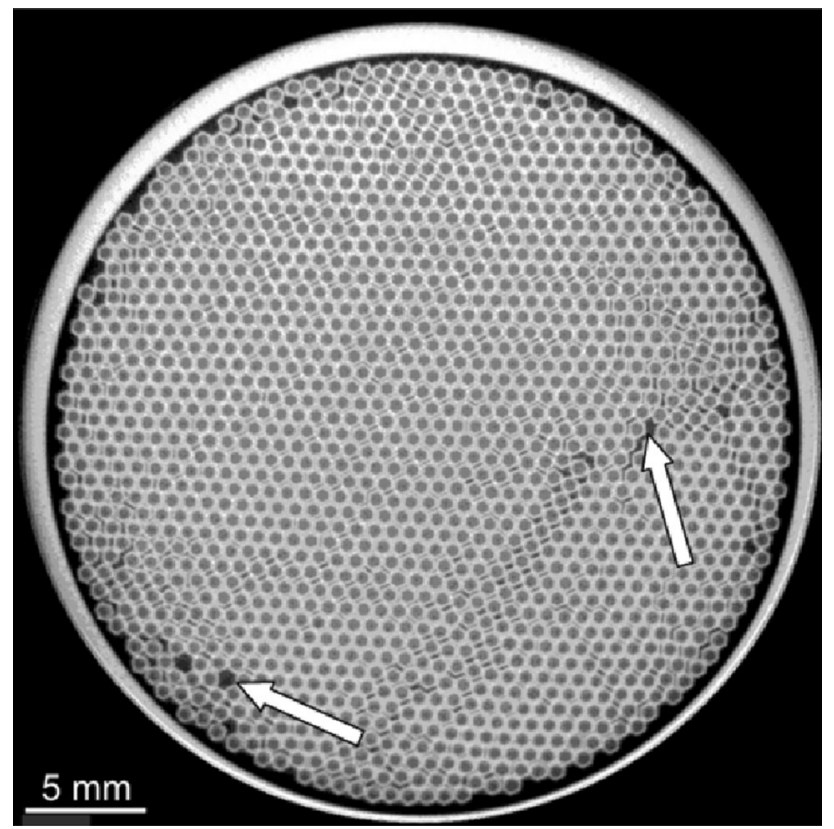

Fig. 7 Cross-section of phantom. Arrows indicate examples of channels and gaps of capillary bundles

structure of phantoms: porosity, surface of channels and capillary bundles, wall thickness of the capillary bundles, equivalent inner diameters (without walls) of channels and capillary bundles, and capillary bundle elongation.
The parameters obtained through the quantification analysis are listed in Table 2 . It is noteworthy that the fine channel share is nearly $8 \%$ of the entire phantom pore space. The bundles of capillaries are characterized by a relatively high uniformity of equivalent diameter, which is nearly 2.7 times greater than that in the case of fine channels. The large standard deviation of channel equivalent diameters indicates their contingency. The structural orientation of the phantom causes the elongation of defects, which confirms a maximum diameter 2 times greater than the minimum diameter of fine channels. Such disturbances may generate disorder diffusion.

Complementary figures of parameters describing the structure of the phantom, showing various percentage ranges of equivalent inner diameter distribution of capillary bundles and channels, are presented in Figs. 8 and 9.

During the fabrication procedure of phantom, the diameter of bundles was designed to be $920 \mu \mathrm{m}$. Microtomography research results indicate, however, that the average value of the inner diameter of bundles is $508 \mu \mathrm{m}$ and standard deviation of this parameter is $14 \mu \mathrm{m}$, whereas the average thickness of walls is $166 \mu \mathrm{m}$ with an SD equal to $18 \mu \mathrm{m}$. In total, the diameter of bundles obtained with the use of microtomography is $840 \mu \mathrm{m}$. Taking into account standard deviation values, the diameter of the bundles can reach $872 \mu \mathrm{m}$. The difference of $48 \mu \mathrm{m}$ between the expected and obtained value (taking into

Table 2 Parameterization of phantom microstructure

\begin{tabular}{|c|c|c|c|c|}
\hline \multirow[t]{2}{*}{ Parameter } & \multirow[t]{2}{*}{ Unit } & \multicolumn{3}{|c|}{ Results of the analysis } \\
\hline & & Entire phantom & Bundles of capillaries & Channels between the walls of the capillary bundles \\
\hline \multicolumn{5}{|l|}{ Global parameters } \\
\hline$V_{\mathrm{v}}$ & $\%$ & 38 & 35 & 3 \\
\hline$S_{\mathrm{v}}$ & $1 / \mathrm{mm}$ & 2.29 & 1.96 & 0.32 \\
\hline$N_{\mathrm{A}}$ & $1 / \mathrm{mm}^{2}$ & 2.85 & 1.98 & 0.87 \\
\hline DA & - & 0.89 & 0.89 & 0.90 \\
\hline \multicolumn{5}{|l|}{ Local parameters } \\
\hline$E(T)$ & $\mu \mathrm{m}$ & - & 166 & - \\
\hline $\mathrm{SD}(T)$ & & & 18 & \\
\hline $\mathrm{CV}(T)$ & & & 0.11 & \\
\hline$E\left(D_{\mathrm{C}}\right)$ & $\mu \mathrm{ms}$ & 437 & 508 & 191 \\
\hline $\mathrm{SD}\left(D_{\mathrm{C}}\right)$ & & 145 & 14 & 77 \\
\hline $\mathrm{CV}\left(D_{\mathrm{C}}\right)$ & & 0.33 & 0.06 & 0.40 \\
\hline$E\left(D_{\max } / D_{\min }\right)$ & - & 1.34 & 1.11 & 2.01 \\
\hline $\mathrm{SD}\left(D_{\max } / D_{\min }\right)$ & & 0.50 & 0.03 & 0.59 \\
\hline $\mathrm{CV}\left(D_{\max } / D_{\min }\right)$ & & 0.37 & 0.02 & 0.29 \\
\hline
\end{tabular}

Structural parameters: $V_{\mathrm{V}}$, the ratio of pore volume to total volume (porosity); $S_{\mathrm{V}}$, the surface of channels and capillary bundles to the total volume of the analyzed area; $N_{\mathrm{A}}$, both the relative multiplicity of channels and/or capillary bundles per unit area; DA, the degree of anisotropy, where for fully isotropic system $\mathrm{DA}=0$, and for the fully anisotropic DA $=1$ (http://www.skyscan.be/); $T$, wall thickness of the capillary bundles; $D_{\mathrm{C}}$, the equivalent inner diameters (without walls) of channels and/or capillary bundles; $D_{\max } / D_{\min }$, the capillary bundle elongation (where $D_{\max }$ is a maximum diameter, and $D_{\min }$ is a minimum diameter). Statistical parameters: $E(x)$, mean value of variable $x ; \mathrm{SD}(x)$, $\operatorname{standard}$ deviation of variable $x ; \mathrm{CV}(x)=\mathrm{SD}(x) / E(x)$ coefficient of variation of variable $x$ 


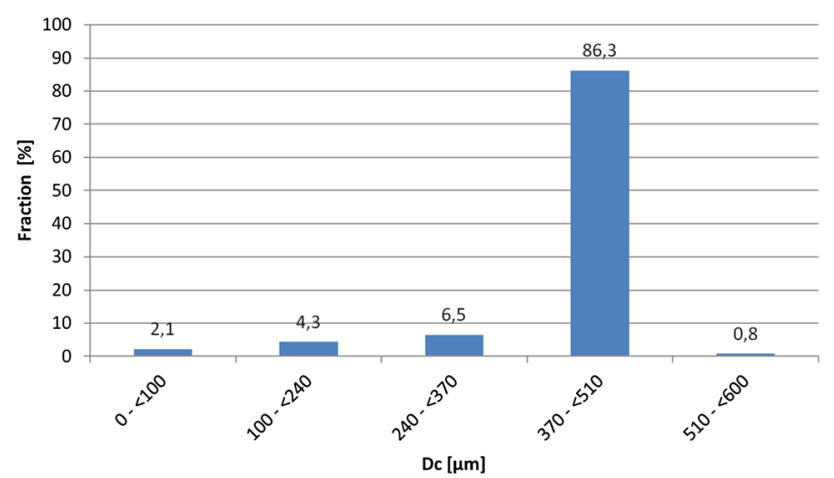

Fig. 8 Total distribution of equivalent inner diameters of capillary bundles and channels for the phantom

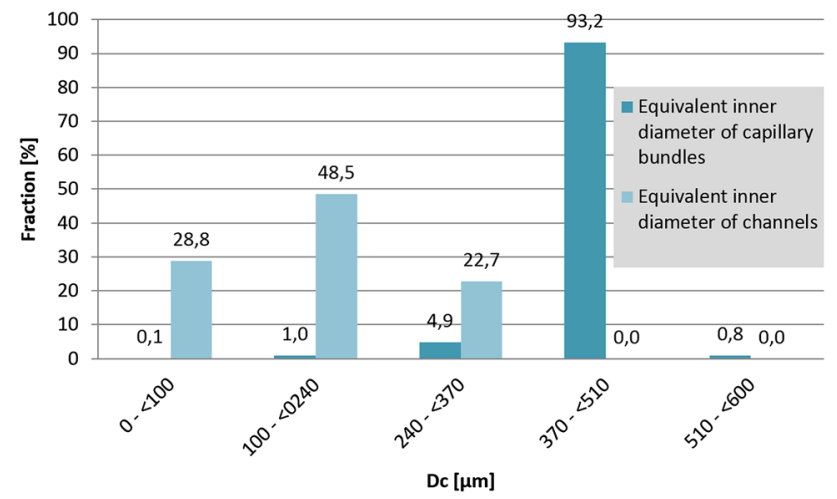

Fig. 9 Phantom: equivalent inner diameter distribution of capillary bundles compared to the equivalent inner diameter of channels

account SD values) might stem from the production process, involving the dense packing of bundles. This fact should be considered in the MRI and DTI studies, due to the relationship between the accuracy of the tests in terms of homogeneity and the linearity of the calibration of the phantom structure. We should also pay attention to the unimodal distribution of capillary bundle diameters, which indicates a favorable distribution of the dominant value closest to the expected one.

The resolution of $\mu \mathrm{CT}$ was not sufficiently good to analyze a singular capillary. This was mainly due to the fact that the samples should not have been broken, thereby precluding individual investigation of individual capillaries. Nevertheless, since the size and geometry of capillary bundles are the result of individual capillaries, the internal structure of phantoms can be characterized by the geometrical relations of bundles.

The next stage of the research was to produce an assessment of homogeneity, which was performed by comparing the area (influence zone, A) obtained by binarization and tessellation image processing transformations. Figure 10 shows the homogeneity assessment process. The results are: $\mathrm{CV}(A) \approx 0.57 ; E(A) \approx 0.30 \mathrm{~mm}^{2} ; \mathrm{SD}(A) \approx 0.17 \mathrm{~mm}^{2}$, where $A$ is the mean size of influence zone. The results of analysis for the bundles of capillaries only are: $\mathrm{CV}(A) \approx 0.04 ; E(A) \approx 0.47 \mathrm{~mm}^{2} ; \mathrm{SD}(A) \approx 0.02 \mathrm{~mm}^{2}$.

Analysis of the obtained results led to an explicit determination of phantom structure and indicated a homogeneity with slight flaws, which should not influence the lateral direction of the diffusion of particles visualized in BST-DTI. The homogeneity of phantom structure determines its applicability as a reference for the comparison of real non-linear capillaries and flow paths of hydrocarbons in rocks.

\section{Fluid flow simulations}

The previous stages, in the case of a cylindrical sample, enabled the visualization of capillary bundles and fine channels. In the next step, we created a three-dimensional numerical model of the cylindrical phantom (Fig. 11) to study fluid flow across the sample. The aim of the

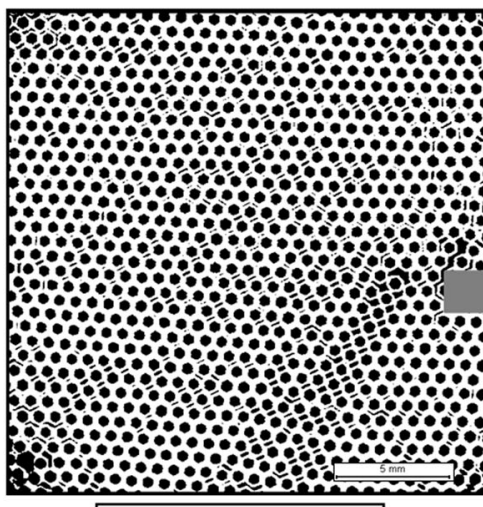

Binarized image

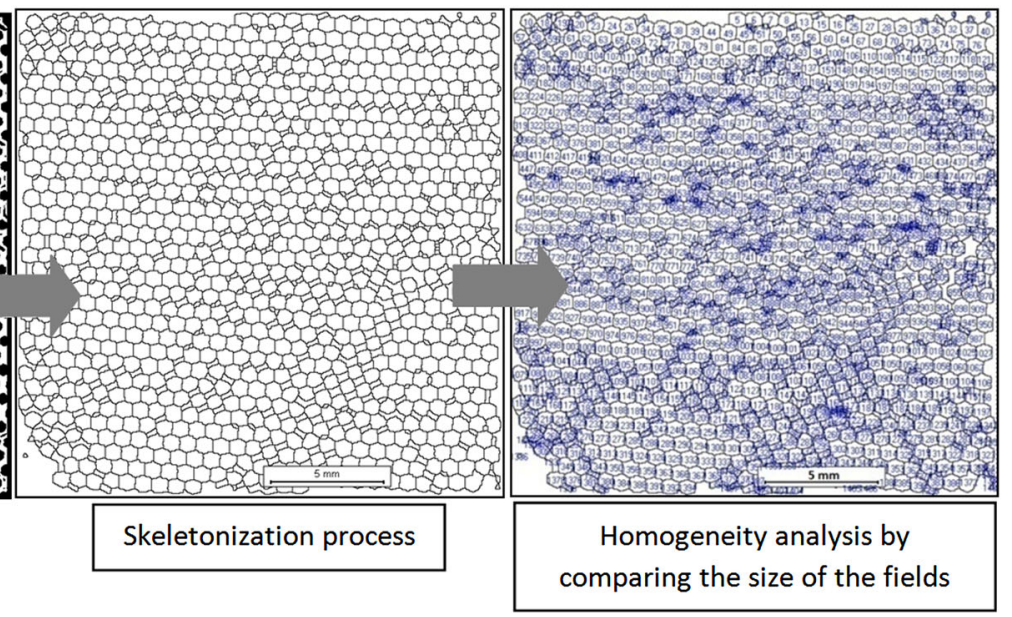

Fig. 10 Transformation process involved in analysis of phantom homogeneity 
Fig. 11 Phantom visualization: a binarized cross-sections; b capillaries without deleted channels
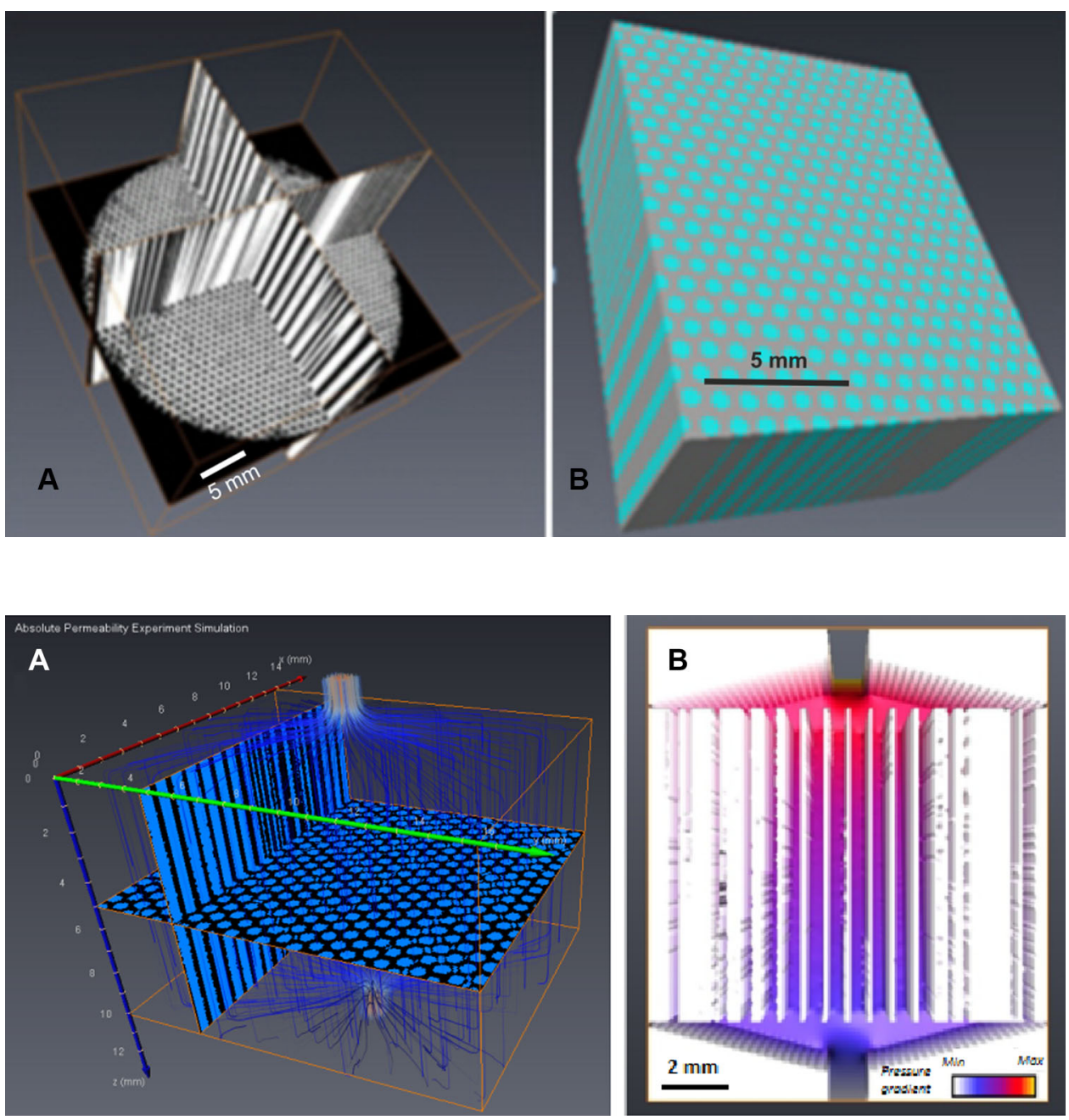

Fig. 12 Visualization of a streamlines, b pressure gradient in phantom simulations was to verify the linearity of the capillaries. In the case of straight, undisturbed and undamaged bundles of capillaries, simulation of fluid flow should result in a linear distribution of streamlines and in a uniform pressure drop in the phantom.

It should be noted that diffusion tensor imaging (DTI) visualizes the movement of hydrogen nuclei. In DTI, anisotropic phantoms are crucial for precise determination of the diffusion tensor components. The resulting visualization of the flow streamlines based on $\mu \mathrm{CT}$ study (Fig. 12) is used to identify potential path movements of nuclei in the DTI method. This approach can be used to verify the impermeability of the materials analyzed, due to the high precision of DTI techniques (even at the nanometric scale). For example, in the case of fractures in the rock samples detected by $\mu \mathrm{CT}$, one can analyze the continuity and propagation of potential media; as in the second step, it is possible to verify the results by DTI. In the case of overlapping streams flow obtained by DTI and by simulation based on $\mu \mathrm{CT}$, the low permeability of the material can be predicted.
As shown in Fig. 12, the linearity of the flow is maintained. Moreover, the centroid path tortuosity for beams of capillaries and channels is 1.015, which also confirms the linearity of the phantom structure. Likewise, uniform pressure changes provide information that there were no larger defects that could interfere with straightforward flow. In the case of contrary results, further more detailed calculation should be performed. The linearity of the sample is the basic criterion that needs to be fulfilled for further proper DTI calibration; therefore, the results obtained indicate the utility of phantoms for MRI scanner calibration. The inhomogeneity of the materials structure, in the form of fine channels and shifts of capillary bundles, revealed during characterization with the use of $\mu \mathrm{CT}$ and light microscope suggested that the linearity of the sample could be disturbed. However, the numerical simulations performed indicate that the sample can be successfully applied in further DTI testing.

Furthermore, there is the question of the influence of capillary walls on flow velocity, which should be studied in future considerations. Another question regards possibly 
scaling up the study of the properties of the reservoir to the global scale. Here, Dvorkin et al. (2009) suggested a positive correlation analysis of reservoir rocks at different scales.

\section{Conclusions}

This study presents universal internal structure parameterization and visualization guideline analysis for BST-DTI calibration phantoms. MRI scanners require a calibration process, which involves determination of the relation between T1, T2 relaxation times distribution, signal magnitudes and sample porosity. Such calibration is possible by the precise definition of DTI phantoms. However, the manufacturing process of the materials can introduce some flaws or discrepancies from the designed geometry, which could seriously skew MRI scanner calibration. The results presented here demonstrate that high-resolution X-ray computed microtomography is an adequate non-destructive technique for the characterization of phantoms designed for rock analysis. The quantitative analysis procedure proposed here serves as a tool for assessing the applicability of phantoms to the application of DTI for the characterization of specific rock structures. According to this procedure, we were able to prove the usefulness of a cylindrical phantom for the calibration of MRI scanners and further in-depth analysis.

The study clearly showed that computed microtomography can be performed without sophisticated treatment of the sample and may provide many parameters that describe the internal structure of phantoms (porosity, inner diameter of capillary bundles). Moreover, the anisotropy of the samples can be described. The must-have factor for this method is good contrast (based on linear coefficient of $\mathrm{X}$-ray absorption) between the liquid and the structure of the phantom. The differences at all stages (acquisition, reconstruction, processing and analysis) show that long acquisition and reconstruction periods are required to obtain higher resolutions. The advantage of $\mu \mathrm{CT}$ is that it delivers the high precision images of internal microstructure, which are used to create three-dimensional visualizations and simulations. The finite volume method proved to be a useful supplementary tool in the analysis of linearity of the sample, when characterization of the material indicated manufacturing flaws. The high-resolution X-ray computed microtomography analysis and numerical modeling with the use of a finite volume method reveal capabilities for complementary use for DTI research technology.

Acknowledgements This work was supported by the National Center for Research and Development (Contract No. PBS2/A2/16/2013).
Open Access This article is distributed under the terms of the Creative Commons Attribution 4.0 International License (http://crea tivecommons.org/licenses/by/4.0/), which permits unrestricted use, distribution, and reproduction in any medium, provided you give appropriate credit to the original author(s) and the source, provide a link to the Creative Commons license, and indicate if changes were made.

\section{References}

Appoloni C, Fernandes C, Rodrigues C (2007) X-ray microtomography study of a sandstone reservoir rock. Nucl Instrum Methods Phys Res Sect A 580:629-632. doi:10.1016/j.nima.2007.05.027

Aurenhammer F (1991) Voronoi diagrams - a survey of a fundamental geometric data structure. ACM Comput Surv 23:345-405. doi: $10.1145 / 116873.116880$

Baker D, Mancini L, Polacci M, Higgins M, Gualda G, Hill R, Rivers M (2012) An introduction to the application of X-ray microtomography to the three-dimensional study of igneous rocks. Lithos 148:262-276. doi:10.1016/j.lithos.2012.06.008

Bielecki J, Jarzyna J, Bożek S, Lekki J, Stachura Z, Kwiatek W (2013) Computed microtomography and numerical study of porous rock samples. Radiat Phys Chem 93:59-66. doi:10.1016/ j.radphyschem.2013.03.050

Callaghan P (1994) Principles of nuclear magnetic resonance microscopy. Oxford University Press, Oxford

Coates GR, Xiao L, Prammer MG (1999) NMR logging principles and applications. Halliburton Energy Services Publication, Houston

Das S (2004) Nuclear magnetic resonance spectroscopy. Resonance 9:34-49

Dvorkin J, Derzhi N, Fang Q, Nur A, Nur B, Grader A, Baldwin C, Tono H, Diaz E (2009) From micro to reservoir scale: permeability from digital experiments. Lead Edge 28:1446-1452. doi:10.1190/1.3272699

Gnudde V, Boone M (2013) High-resolution X-ray computed tomography in geosciences: a review of the current technology and applications. Earth Sci Rev 123:1-17. doi:10.1016/j.ear scirev.2013.04.003

Kaczmarek Ł, Maksimczuk M, Wejrzanowski T, Krzyżak A (2015) High-resolution X-ray microtomography and nuclear magnetic resonance study of a carbonate reservoir rock. In: Proc. 15th International Multidisciplinary Scientific GeoConference SGEM 2015, June 18-24, 2015, Albena. doi:10.5593/SGEM2015/B11/ S6.099

Ketcham R, Carlson W (2001) Acquisition, optimization and interpretation of X-ray computed tomographic imagery: applications to the geosciences. Comput Geosci 27:381-400. doi:10. 1016/S0098-3004(00)00116-3

Komlosh ME, Özarslan E, Lizak MJ, Horkay F, Schram V, Shemesh N, Cohen Y, Basser PJ (2011) Pore diameter mapping using double pulsed-field gradient MRI and its validation using a novel glass capillary array phantom. J Magn Reson 208:128-135. doi:10.1016/j.jmr.2010.10.014

Krzyżak AT, Olejniczak Z (2014) Improving the accuracy of PGSE DTI experiments using the spatial distribution of $b$ matrix. Magn Reson Imaging 33:286-295. doi:10.1016/j.mri.2014.10.007

Krzyżak AT, Jasiński A, Adamek D (2005) Qualification of the most statistically "sensitive" diffusion tensor imaging parameters for detection of spinal cord injury. Acta Phys Pol Ser A 108:207-210

Krzyżak AT, Jasiński A, Kwieciński S, Kozłowski P, Adamek D (2008) Quantitative assessment of injury in rats spinal cord in vivo using MRI of water diffusion tensor. Appl Magn Reson 34:1-24. doi:10.1007/s00723-008-0095-7 
Krzyżak AT, Kłodowski K, Raszewski Z (2015) Anisotropic phantoms in magnetic resonance imaging. In: Proc. 37th Annual International Conference of the IEEE Engineering in Medicine and Biology Society, August 25-29, 2015, MiCo, Milano

Petchsingto T, Karpyn T (2009) Deterministic modeling of fluid flow through a CT-scanned fracture using computational fluid dynamic. Energy Sources Part A Recovery Util Environ Effects 31:897-905. doi:10.1080/15567030701752842

Straley C, Rossini D, Vinegar H, Tutunjian P, Morriss C (1995) Core analysis by low field NMR. SCA-9404, pp 43-56

Sun Q, Xue L, Zhu S (2015) Permeability evolution and rock brittle failure. Acta Geophys 63(4):978-999. doi:10.1515/acgeo-20150017

Tomanek B, Jasiñski A, Sulek Z, Muszyñska J, Kulinowski P, Kwieciński S, Krzyżak A, Skórka T, Kibiński J (1996) Magnetic resonance microscopy of internal structure drone and queen honey bees. J Apic Res 35:3-9. doi:10.1080/00218839.1996. 11100907
Twarduś E, Nowicka A (2014) Dokumentacja wynikowa otworu badawczego Opalino. PGNIG, Piła

Wei D-F, Liu X-P, Hu X-X, Xu R, Zhu L-L (2015) Estimation of permeability from NMR logs based on formation classification method in tight gas sands. Acta Geophys 63(5):1316-1338. doi:10.1515/acgeo-2015-0042

Wejrzanowski T, Spychalski WL, Rożniatowski K, Kurzydłowski KJ (2008) Image based analysis of complex microstructures of engineering materials. Int J Appl Math Comput Sci 18:33-39. doi:10.2478/v10006-008-0003-1

Xiao L, Zou C, Mao Z, Shi Y, Liu XP, Jin Y, Guo H, Hu X (2013) Estimation of water saturation from nuclear magnetic resonance (NMR) and conventional logs in low permeability sandstone reservoirs. J Petrol Sci Eng 108:40-51. doi:10.1016/j.petrol. 2013.05.009 